\title{
BMJ Open Qualitative study of primary care clinicians' views on point-of-care testing for C-reactive protein for acute respiratory tract infections in family medicine
}

\author{
Victoria Hardy, ${ }^{1}$ Matthew Thompson, ${ }^{1}$ Gina A Keppel, ${ }^{1}$ William Alto, ${ }^{2}$ \\ M Ashworth Dirac, ${ }^{3}$ Jon Neher, ${ }^{4}$ Christopher Sanford, ${ }^{1}$ Jaime Hornecker, ${ }^{5}$ \\ Allison Cole ${ }^{1}$
}

To cite: Hardy V,

Thompson M, Keppel GA, et al. Qualitative study of primary care clinicians' views on point-of-care testing for C-reactive protein for acute respiratory tract infections in family medicine. BMJ Open 2017;7:e012503

doi:10.1136/bmjopen-2016012503

- Prepublication history and additional material is available. To view please visit the journal (http://dx.doi.org/ 10.1136/bmjopen-2016012503).

Received 3 May 2016 Revised 22 November 2016 Accepted 22 December 2016

CrossMark

For numbered affiliations see end of article.

Correspondence to

Victoria Hardy;

vhardy4@uw.edu

\section{ABSTRACT}

Objective: To explore clinicians views of the barriers and facilitators to use of $\mathrm{C}$-reactive protein (CRP) point-of-care tests (POCT) in US family medicine clinics for the management of acute respiratory tract infections (ARTIs) in adults.

Setting: Five family medicine clinics across two US states.

Participants: 30 clinicians including 18 physicians, 9 physician residents, 2 physician assistants and 1 nurse practitioner, took part in the study.

Design: A qualitative study using a grounded theory approach to thematically analyse focus group interviews.

Results: These clinicians had limited access to diagnostic tests for patients with ARTI, and very little knowledge of CRP POCT. Three major themes were identified and included the potential clinical role of CRP POCT, concerns related to implementing CRP POCT and evidence needed prior to wider adoption in family medicine. Clinicians believed CRP POCT could support decision-making for some presentations of ARTIs and patient populations when used in conjunction with clinical criteria. Clinicians had concerns about possible overuse and inaccuracy of CRP POCT which they believed might increase antibiotic prescribing rates. Other concerns identified included integration of the test with clinic workflows and cost-effectiveness.

Conclusions: Clinicians stand at the forefront of antibiotic stewardship efforts, but have few diagnostic tests to help them confidently manage ARTIs. CRP POCT may facilitate some aspects of clinical practice. Incorporating CRP POCT with clinical guidelines may strengthen utility of this test, when there is diagnostic uncertainty.

\section{INTRODUCTION}

Acute respiratory tract infections (ARTIs) are one of the most common reasons for adults

\section{Strengths and limitations of this study}

- This is the first study to explore clinician attitudes towards $C$ reactive protein point-of-care tests (CRP POCT) for acute respiratory infections in adults in US primary care settings.

- Potential barriers and facilitators of future implementation of CRP POCT in the USA related to clinical utility of this test are highlighted, as well as organisational and financial arrangements that need to be addressed in order for CRP POCT to be integrated into clinical practice and family medicine clinics.

- Clinicians had limited knowledge and experience of CRP POCT, in any type of healthcare setting. Consequently, attitudes presented in this study may have been influenced by the description of CRP POCT given by interviewers, rather than from working knowledge of the test.

presenting to primary care settings in the USA. ${ }^{12}$ For ARTIs such as acute bronchitis, sinusitis and pharyngitis that are predominantly viral, antibiotics confer little or no benefit for reducing risks of serious complications, nor significantly reduce time to symptom resolution. ${ }^{3}$ Yet reportedly between 2007 and $2009, \sim 51 \%$ of adult patients were prescribed antibiotics unnecessarily for these types of ARTIs ${ }^{4}$ and more recently $67.8 \%$ of patients presenting to outpatient settings were reported as using antibiotics for acute bronchitis. ${ }^{5}$ Diagnostic and prognostic uncertainties, due to overlapping signs and symptoms between bacterial and viral infections $^{6}$ and perceived patient demand for antibiotics, have been identified as drivers of inappropriate antibiotic prescribing. ${ }^{7-9}$ Overuse of antibiotics is associated with the emergence of antibiotic-resistant microorganisms, 
which can cause treatment failure, ${ }^{10}$ and heighten risk of future adverse events. ${ }^{11}$ Escalating prevalence of antimicrobial resistance coupled with slow development of new antibiotics ${ }^{12}$ means identifying interventions to encourage judicious prescribing is a national and global priority.

Over the last 20 years, there have been numerous attempts in the USA to reduce inappropriate prescribing; these include the Centers for Disease Control and Prevention's (CDC) 'Get Smart: Know When Antibiotics Work' initiative in $1995^{13}$ and more recently the 'Choosing Wisely' campaign from the American Board of Internal Medicine Foundation. ${ }^{14}$ While reduced prescribing rates have been reported for children, antibiotic use has remained static in adults and actually increased in older adult populations ( $\geq 65$ years). ${ }^{15}$ C-reactive protein (CRP) is a biomarker of systemic inflammation that quantifies serum CRP from a finger-prick blood sample within 4 min, ${ }^{16}$ with levels typically more elevated during bacterial than viral infections. ${ }^{17}$ CRP point-of-care tests (POCT) used as part of the clinical assessment for ARTI may help clinicians better discern patients with bacterial aetiology, and thus which patients would benefit from antibiotics. ${ }^{18} 19$ Robust evidence from two meta-analyses found that CRP POCT was significantly associated with reduced antibiotic prescribing for ARTIs at the initial patient visit. ${ }^{20}{ }^{21}$ CRP POCT is used routinely in clinical practice in some European countries, but is not widely used in family medicine in the USA. ${ }^{22}$ In this qualitative study, we explore the potential acceptability to family medicine clinicians of using CRP POCT as part of the clinical workup for adults with ARTIs in US primary care.

\section{METHODS}

Subjects

Family medicine clinicians were recruited from five clinics in the Washington, Wyoming, Alaska, Montana and Idaho (WWAMI) region Practice and Research Network (WPRN). The WPRN is a network of over 50 community-based primary care practices across the five-state WWAMI region. Participants were recruited on convenience (either in person or via email) on behalf of the research team, by clinician research champions whose role is to engage clinic staff in WPRN research activities at each site. Clinics were selected based on type (eg, community health centres and hospital-affiliated), location (eg, urban, rural) and clinical interest, to recruit clinicians with a broad range of experiences and perspectives. Participants received written information and gave verbal consent before discussions began. As an early review of the literature had revealed no US-based studies on CRP POCT in US family medicine, the interviewer(s) gave a brief description of CRP POCT and the primary condition of interest (ie, ARTIs) to familiarise participants (see online supplementary file 1). The study proposal was reviewed by the University of
Washington's Human Subjects Division Institutional Review Board and given an exempt determination (reference 49929).

\section{Focus groups}

Audio-recorded focus groups lasting 40-50 min were conducted with participants at each clinic by a family physician (MT) or a non-clinical researcher (VH). Both interviewers' research background is in the use of diagnostic to improve the recognition of infectious diseases. A topic guide developed by the research team was used to guide discussions. Semistructured questions explored opinions about using on-site diagnostic tests to guide antibiotic prescribing, prior experience or knowledge of CRP POCT and attitudes towards use of CRP POCT for adults with suspected ARTIs. Topic guides were informed by literature relating to general use of POCT in primary care, ${ }^{23}$ with a specific focus on CRP POCT for ARTIs, ${ }^{24}$ and were reviewed and revised following input from our research champions prior to use. Further questions were added to the topic guide as themes emerged from successive focus groups (see online supplementary file 1). At the end of each focus group, participants completed a brief anonymous survey to provide descriptive characteristics. Data collection took place between August and December 2015.

\section{Data analysis}

Data analysis began after the first interview had been transcribed. Transcripts were initially read to gain an overall impression of subjects' attitudes, and codes manually annotated in margins. Transcripts were then examined and reflected on to challenge initial data interpretation, and to more formally synthesise themes and subthemes to which segments of text were assigned. Using the constant comparison method influenced by grounded theory, themes were derived inductively and data were cyclically contrasted for 'fit'. Relationships between themes were validated or modified as interviews progressed. ${ }^{25}$ A coding framework was developed by one author (VH), discussed and revised by another (MT) and reviewed by all authors to verify description and grouping of themes reflected the data; minor modifications were made based on feedback from a third author (JN). Qualitative software, Nvivo V.10, was used to facilitate data organisation.

\section{RESULTS}

A total of 30 clinicians participated from the five clinics. Eighteen $(58 \%)$ were family physicians, nine $(31 \%)$ family physician residents, as well as two physician assistants and one nurse practitioner (one family physician did not complete the written survey due to time constraints) (table 1). Three major themes were identified: (1) potential clinical role of CRP POCT; (2) concerns related to implementation and (3) evidence needed prior to use of CRP POCT in family medicine clinics, 
Table 1 Participant characteristics, $\mathrm{N}=30$

\begin{tabular}{lc}
\hline Characteristics & $\mathbf{n}(\%)$ \\
\hline Type of practitioner & \\
Faculty/staff physician & $18(58.6)$ \\
Resident physician & $9(31)$ \\
Physician assistant & $2(6.9)$ \\
Nurse practitioner & $1(3.4)$ \\
Gender & $16(55.2)^{\star}$ \\
$\quad$ Male, $\mathrm{n}(\%)$ & $46.9(27-70)^{\star}$ \\
Mean age in years (range) & $8.6(0.08-38)^{\star}$ \\
Mean years at clinic (range) & \\
"Excludes descriptive data for one family physician who did not \\
complete the survey.
\end{tabular}

discussed below. Additional quotations supporting themes are provided in online supplementary file 2 (quotes are protected using participant $(\mathrm{C}) /$ site codes $(\mathrm{S})$ )

Most participants had experience of using CRP as a send-out laboratory test for rheumatological conditions and suspected sepsis, but few had knowledge or experience of CRP POCT for the workup of ARTIs, in any healthcare setting. Participants used rapid group A streptococcal tests to guide antibiotic prescribing for sore throat and some had also used procalcitonin for suspected pneumonia and sepsis in inpatient settings. Other diagnostic tests (including send out) for ARTIs in adults were reported as being rarely used, although occasionally chest radiography and white cell count were used.

\section{Potential clinical role(s) of point-of-care CRP}

Participants mostly described feeling confident knowing when to prescribe antibiotics, and equally when to withhold them, when patients' infections (or their perception that it was severe or bacterial) could be clearly dichotomised into present or absent. However, they identified a need for diagnostic assistance in the "middle cases" (P5, S3) where aetiology is not reliably discerned from patients' symptoms, or where there is uncertainty about the 'window of opportunity' in the time course of a patient's illness within which an antibiotic would be beneficial, "like that sinus infection at six and a half days, where you're not sure" (P3, S5), and for which "guidelines fail me" (P3, S2). Participants also described diagnostic uncertainty for patients at increased risk of complications, such as those with acute infective exacerbations of chronic obstructive pulmonary disease and asthma; scenarios which were believed to more likely result in precautionary antibiotic prescribing for patient safety reasons (Q1), and for which they noted that CRP POCT could play a role in clinical practice.

Generally, participants believed expectations for antibiotics had abated over recent years (Q2), with two noting that patients seem relieved "that I [the provider] am not just turning to antibiotics" (P4, S1). However, clinicians continued to perceive pressure for antibiotics from a subgroup of patients, including those with whom clinicians did not have a relationship, parents of young children or patients whom they believed had been given antibiotics unnecessarily in the past for similar (selflimiting) illnesses. For these patients, not prescribing was described as sometimes more difficult and time consuming than simply prescribing (Q3). Having a visible test result to share with unconvinced patients during the patient visit might reassure patients that clinicians were making appropriate treatment decisions, "because sometimes I think they think we are just guessing" (P4, S1). Equally, a test such as this was viewed as affording an opportunity to share the justification underpinning antibiotic prescribing decisions with patients, which might make them aware of the "complexity and lack of clarity about medical decision-making", that "doctoring is hard" (P3, S1), and understand that a "no prescribing decision' does not mean their concerns are being dismissed (Q4). Therefore, for "select patients it [CRP POCT] could be a valuable tool” (P3, S5).

\section{Concerns related to implementation}

Participants expressed concerns about the risk of falsely elevated results in patients with underlying inflammatory conditions and for whom CRP POCT might not be appropriate to use (eg, rheumatoid arthritis) (Q5), and the consequences of getting a false negative result in the presence of serious bacterial infections (Q6). High false positive rates for bacterial aetiology could increase antibiotic prescribing, especially as "clinics become more efficient, then all of a sudden the test is being done automatically" (Q7). "Having the patient present with this [respiratory complaint] and the MA [medical assistant] just does the CRP"(P2, S3) before the patient has been clinically assessed may pressurise clinicians into treating with antibiotics in situations where they later believe the result to be falsely positive, increasing antibiotic prescribing rates (Q8). Participants emphasised CRP POCT should supplement clinical findings: "We should be using our clinical skills to come up with what we think is the right diagnosis. We should only be using the test to confirm or refute the diagnosis" (P1, S3). Not giving more precedence to CRP POCT over time might be challenging and resident physicians were worried they might come to depend on the test to the detriment of developing clinical skills. A small number of participants identified the risk of medicalising self-limiting illnesses through unnecessary use of CRP POCT which they believed might increase patient demand for diagnostic testing in future encounters (Q9).

There were mixed opinions about how CRP POCT could be integrated into clinic workflows. Participants at one clinic had concerns about the time that would be needed to administer and interpret the test, primarily related to clinics' testing processes (Q10). For example, POCTs that are conducted in on-site laboratories, involve the patient leaving the examination room to get 
the test and then returning. Participants at one clinic viewed this as an impediment to implementation because "it changes a 15-minute encounter into a 55-minute process, which is a disincentive to get any diagnostic test" (P3, S5). In contrast, others believed if it "saved counselling people out of antibiotics" (P6, S3) then it could save time.

Without knowing reimbursement rates and patient out-of-pocket costs of CRP POCT which were noted to vary depending on the types of insurance coverage at clinics (ie, private insurance, Medicare/Medicaid) as well as overall cost-effectiveness, participants were uncertain about the financial viability of using CRP POCT (Q11). One clinic which subsidised the cost of antibiotics at their pharmacy, felt use of CRP POCT could offset the high costs of some antibiotics (Q12).

\section{Evidence needed prior to implementing}

Participants held mixed views on the overall impact of CRP POCT on antibiotic prescribing rates, which were linked to clinicians' general understanding of CRP, perceived discriminatory value, beliefs about the helpfulness of CRP POCT for counselling patients and how the test would be used (or overused). Some believed "it would $[\ldots]$ without a doubt lower antibiotic prescribing" (P3, S2) while others were "not sure the CRP test would decrease the number of antibiotic prescriptions" (P2, $\mathrm{S5}$ ). Therefore, evidence related to the clinical effectiveness of CRP POCT in reducing antibiotic use "[if] on a national or large level [CRP POCT] brought down antibiotic use. That would be really alluring" (P2, S5), was desired. Participants described the need for data from robustly designed studies, "RCTs would be best" (P3, S3). There was a particular desire for clinical studies that are generalisable to US populations (Q15), because "you can't compare apples to oranges when you're dealing with a study in Scandinavia where they don't have diabetes, they're not homeless" (P4, S2). Additionally, diagnostic accuracy information showing false positive and false negative rates of CRP POCT and CRP levels in bacterial and viral infections, including how quickly levels become elevated (and how high and for what duration) during the course of ARTI, would be beneficial to help them interpret test results (Q13; Q14). Finally, data that could be applied to Native American populations who they considered at higher risk of complications from ARTIs and for whom clinicians "treat outside the typical window" (P3, S2) were identified as a need.

\section{DISCUSSION}

Key findings

Family medicine clinicians currently have a limited number of diagnostic tests in their arsenal to help discriminate between bacterial and viral ARTIs in adults. Despite convincing evidence supporting the use of CRP POCT for ARTIs in primary care settings, and their routine clinical use in many European countries, ${ }^{26}$ surprisingly few clinicians had knowledge of this test, and most had never used CRP POCT in primary or secondary care. Overall, CRP POCT was acceptable to clinicians who believed for defined patient populations and clinical scenarios (eg, where there was lingering uncertainty, perceived patient pressure or for patients at elevated risk of developing complications), CRP POCT if used as an adjunct to clinical assessment, could be used to support patient counselling efforts, and improve antibiotic prescribing decisions for ARTIs; although they were undecided about the overall impact of CRP POCT on national prescribing rates. Clinicians were concerned about inaccuracy and usefulness of CRP POCT in the context of patients with inflammatory comorbidities who are more likely to falsely test positive, overprescribing precipitated by overtesting leading to undermining of clinicians' skills and encouraging future patient demand for diagnostic tests and cost-effectiveness.

\section{Comparison with existing literature}

A systematic review of qualitative studies (none from the USA) on clinicians' perceptions of POCT blood testing (eg, CRP, haemoglobin A1c) in primary care, identified similar findings to ours specific to CRP POCT, including improved confidence in decision-making in certain clinical scenarios, worry of inaccurate results and concern over-reliance on diagnostic tests might undermine clinician's clinical skills. ${ }^{23}$ Also consistent with our study, Jones et al reinforce the diagnostic need for discerning bacterial from viral aetiology, particularly in situations where signs and symptoms fail to provide sufficient discriminatory value. As CRP values at extreme ends of the spectrum typically allow aetiology to be better discerned $^{27}$ than do values that are intermediately elevated, ${ }^{28}$ reconciling this diagnostic need with the potential uncertainty of CRP as a marker for patients at intermediate risk of adverse outcomes, needs to be balanced. While some diagnostic uncertainty in clinical practice may be unavoidable, ${ }^{27}$ in these scenarios diagnostic certainty might be enhanced in the USA by incorporating CRP POCT into clinical guidelines (such as the National Institute for Health and Care Excellence (NICE) guidelines for pneumonia in the $\mathrm{UK}^{22}$ ), that recommend when CRP POCT is appropriate, as well as the threshold value/range indicating rational prescribing of antibiotics. Indeed, use of CRP POCT in conjunction with the clinical assessment for the diagnostic workup of ARTIs, ${ }^{29}$ and for enhancing patient communication ${ }^{16}$ has been expressed by clinicians elsewhere in the literature, as well as in our findings. Although not significantly changing the proportion of patients falling into the intermediate-risk category, adding CRP POCT to the clinical assessment has been reported to improve accurate discrimination of patients with and without pneumonia (ie, increased true positive and negative results), potentially improving the reliability of diagnostic information available to clinicians. ${ }^{30}$ 
Additionally, in many family medicine clinics, MAs are the first person to see the patient. With more POCTs being given a Clinical Laboratory Improvement Amendment (CLIA)-waived determination (ie, limited complexity to implement/interpret and low risk of erroneous results) (eg, Rapid Strep), ${ }^{31}$ MAs may be poised to undertake more of this type of testing in the future. Our findings underscore a lack of clarity about how delivery of CRP POCT would be optimised in family medicine clinics. Mixed views about how CRP POCT (and POCT more generally) would be integrated into clinic workflows, specifically, who will perform the testing and when, coincides with existing research. ${ }^{16}{ }^{32}$ Uncertainty about the cost-effectiveness of POCT has been repeatedly cited as a constraint to broader implementation, supported by our findings. ${ }^{33} 34$

\section{Strengths and limitations of the study}

This is the first study to explore US clinician's knowledge and attitudes about use of POCT CRP for clinical management of ARTI, which is relevant to stakeholders nationally for considering the role existing diagnostic technologies could play in strengthening frontline antibiotic stewardship efforts. We collected data from clinicians at different types of family medicine clinics, which broadened the scope of issues elicited. Data collection ended after the fifth focus group due to thematic saturation, which is consistent with qualitative standards when using focus groups for data collection with homogenous participants. ${ }^{35}$ Focus groups were facilitated by a family physician with experience evaluating diagnostic tests for infectious diseases. To ensure discussions were balanced and guided by emergent issues, a second (non-clinical) researcher was present for four of the focus groups, and also led data analysis. As most clinicians had limited knowledge of CRP POCT, attitudes could have been influenced by the description given by interviewers. Although care was taken not to present opinions/knowledge biasing responses, different attitudes might have been provoked if participants had first-hand experience of this test.

\section{Implications for clinicians, researchers and industry}

Although the repertoire of diagnostic tests available to clinicians is expanding, relatively few POCT appear to be routinely used in family medicine to guide antibiotic prescribing decisions for ARTIs. Antibiotic stewardship efforts need to be multifaceted (eg, encompassing public education strategies, clinician counselling skills), but our study suggests there remains an unmet diagnostic need ${ }^{36}$ for a wider range of POCT to be added to the diagnostic workup of ARTIs in the USA to facilitate the safe reduction of antibiotics for infections not enabling a clear 'rule in/out' decision. Recognising this perennial need, a White House report has endorsed prioritising the development and implementation of point-of-care diagnostic technologies that facilitate better identification of infectious agents to enable pathogen-targeted treatment, further reducing unnecessary antibiotic prescribing; a strategy that has been promoted and incentivised internationally (ie, the Longitude Prize). ${ }^{37} 38 \mathrm{In}$ the USA, this will require a dialogue between technology developers, researchers and clinician stakeholders to ensure the features of POCT are harnessed to fulfil clinical needs ${ }^{39}$; evaluating the diagnostic and prognostic value of existing technologies (including CRP POCT) through rigorous clinical trials ${ }^{40}$ that include populations such as American Indians and Alaskan Natives with greater risk of hospitalisation and mortality from ARTIs than the general US population, ${ }^{41}$ and who are not currently represented in existing European trial data; collaborating with stakeholders to develop an implementation plan assisting integration into clinical practice, ${ }^{39}$ and determining tests' cost-effectiveness. As CRP results cannot be dichotomised, ambiguity ruling out infections in diagnostic grey areas may persist without clear guidance for clinicians on how to assimilate CRP POCT results with clinical assessment findings in a meaningful way. Understanding the added information provided by CRP POCT results in scenarios where there is diagnostic uncertainty, could enhance utility of this test. ${ }^{30}$

\section{CONCLUSION}

CRP POCT was acceptable to clinicians for supporting decision-making related to ARTIs in certain clinical scenarios and patient populations. To improve diagnostic certainty and assuage clinicians' concerns regarding the effects of unnecessary testing and potential inaccuracy, utility of CRP POCT in US family medicine may be strengthened by clinical guidelines indicating when CRP POCT is indicated and antibiotic therapy justified. Determining how to integrate this test into clinic workflows along with cost-effectiveness will also be important for wider adoption in the USA.

\section{Author affiliations}

${ }^{1}$ Department of Family Medicine, University of Washington, Seattle, Washington, USA

${ }^{2}$ Seattle Indian Health Board, Seattle, Washington, USA

${ }^{3}$ Swedish Family Medicine First Hill, Seattle, Washington, USA

${ }^{4}$ Valley Family Medicine, Renton, Washington, USA

${ }^{5}$ Family Medicine Residency, University of Wyoming, Casper, Wyoming, USA

Acknowledgements This study was funded by an unrestricted grant from Alere. The WWAMI region Practice and Research Network is supported by the National Center for Advancing Translational Sciences of the National Institutes of Health under Award Number UL1TR000423. We thank the clinicians who participated in this study.

Contributors MT conceived and designed the study; MT and VH collected the data; VH and MT analysed the data. VH drafted and MT revised the manuscript. GAK, MAD, WA, JN and CS contributed to development of the manuscript. All authors reviewed and commented on the manuscript.

Funding Alere Inc.

Disclaimer The content is solely the responsibility of the authors and does not necessarily represent the official views of the funders of this study or of the National Institutes of Health. 
Competing interests MT has received funding from Roche Molecular Diagnostics for consultancy work, and funding from the Patient-Centered Outcomes Research Institute for research on diagnostic test methods. The other authors declare no competing interests.

Ethics approval University of Washington Human Subject's Division.

Provenance and peer review Not commissioned; externally peer reviewed.

Data sharing statement No additional data are available.

Open Access This is an Open Access article distributed in accordance with the Creative Commons Attribution Non Commercial (CC BY-NC 4.0) license, which permits others to distribute, remix, adapt, build upon this work noncommercially, and license their derivative works on different terms, provided the original work is properly cited and the use is non-commercial. See: http:// creativecommons.org/licenses/by-nc/4.0/

\section{REFERENCES}

1. Gonzales R, Anderer T, McCulloch CE. A cluster randomized trial of decision support strategies for reducing antibiotic use in acute bronchitis. JAMA Intern Med 2013;173:267-73.

2. Harris AM, Hicks LA, Qaseem A, High Value Care Task Force of the American College of Physicians and for the Centers for Disease Control and Prevention. Appropriate antibiotic use for acute respiratory tract infection in adults: advice for high-value care from the American College of Physicians and the Centers for Disease Control and Prevention. Ann Intern Med 2016;164:425-34.

3. Smith SM, Fahey T, Smucny J, et al. Antibiotics for acute bronchitis. Cochrane Database Syst Rev 2014(3):CD000245

4. Shapiro DJ, Hicks LA, Pavia AT, et al. Antibiotic prescribing for adults in ambulatory care in the USA, 2007-09. J Antimicrob Chemother 2014;69:234-40.

5. Ebell MH, Radke T. Antibiotic use for viral acute respiratory tract infections remains common. Am J Manag Care 2015;21:e567-575.

6. Horwood J, Cabral C, Hay AD, et al. Primary care clinician antibiotic prescribing decisions in consultations for children with RTIs: a qualitative interview study. Br J Gen Pract 2016;66:e207-13

7. Whaley LE, Businger AC, Dempsey PP, et al. Visit complexity, diagnostic uncertainty, and antibiotic prescribing for acute cough in primary care: a retrospective study. BMC Fam Pract 2013;14:120.

8. Little P, Dorward M, Warner G, et al. Importance of patient pressure and perceived pressure and perceived medical need for investigations, referral, and prescribing in primary care: nested observational study. BMJ 2004;328:444.

9. Jakobsen KA, Melbye H, Kelly MJ, et al. Influence of CRP testing and clinical findings on antibiotic prescribing in adults presenting with acute cough in primary care. Scand $J$ Prim Health Care 2010;28:229-36.

10. Garau J, Nicolau DP, Wullt B, et al. Antibiotic stewardship challenges in the management of community-acquired infections for prevention of escalating antibiotic resistance. J Glob Antimicrob Resist 2014;2:245-53.

11. Llor C, Bjerrum L. Antimicrobial resistance: risk associated with antibiotic overuse and initiatives to reduce the problem. Ther Adv Drug Saf 2014:5:229-41.

12. Bartlett JG, Gilbert DN, Spellberg B. Seven ways to preserve the miracle of antibiotics. Clin Infect Dis 2013;56:1445-50.

13. Centers for Disease Control and Prevention. Get Smart: know when antibiotics work. http://www.cdc.gov/getsmart/campaign-materials/ about-campaign.html (accessed 20 Jul 2013).

14. Foundation A. An initiative of the $A B M$ foundation. Choosing Wisely, 2013.

15. Lee GC, Reveles KR, Attridge RT, et al. Outpatient antibiotic prescribing in the United States: 2000 to 2010. BMC Med 2014;12:96.

16. Huddy JR, Ni MZ, Barlow J, et al. Point-of-care $\mathrm{C}$ reactive protein for the diagnosis of lower respiratory tract infection in NHS primary care: a qualitative study of barriers and facilitators to adoption. BMJ Open 2016:6:e009959.

17. Haran JP, Beaudoin FL, Suner S, et al. C-reactive protein as predictor of bacterial infection among patients with an influenza-like illness. Am J Emerg Med 2013;31:137-44.
18. Cals JW, Schot MJ, de Jong SA, et al. Point-of-care C-reactive protein testing and antibiotic prescribing for respiratory tract infections: a randomized controlled trial. Ann Fam Med 2010;8:124-33.

19. Biomarkers as point-of-care tests to guide prescription of antibiotics in patients with acute respiratory infections in primary care. 2014.

20. Huang Y, Chen R, Wu T, et al. Association between point-of-care CRP testing and antibiotic prescribing in respiratory tract infections: a systematic review and meta-analysis of primary care studies. Br J Gen Pract 2013;63:e787-94.

21. Aabenhus R, Jensen JU, Jørgensen KJ, et al. Biomarkers as point-of-care tests to guide prescription of antibiotics in patients with acute respiratory infections in primary care. Cochrane Database Syst Rev 2014;(11):CD010130.

22. (NICE) NIfHaCE. Pneumonia: diagnosis and management of community-and hospital-acquired pneumonia in adults (draft guidance). 2014.

23. Jones CH, Howick J, Roberts NW, et al. Primary care clinicians' attitudes towards point-of-care blood testing: a systematic review of qualitative studies. BMC Fam Pract 2013;14:117.

24. Andreeva $\mathrm{E}$, Melbye $\mathrm{H}$. Usefulness of $\mathrm{C}$-reactive protein testing in acute cough/respiratory tract infection: an open cluster-randomized clinical trial with C-reactive protein testing in the intervention group. BMC Fam Pract 2014;15:80.

25. Glaser BG. The constant comparative method of qualitative analysis. Soc Probl 1965;12:436-45.

26. Cooke J, Butler C, Hopstaken R, et al. Narrative review of primary care point-of-care testing (POCT) and antibacterial use in respiratory tract infection (RTI). BMJ Open Respir Res 2015;2:e000086.

27. Shinkins B, Perera R. Diagnostic uncertainty: dichotomies are not the answer. Br J Gen Pract 2013;63:122-3.

28. Van den Bruel A, Thompson MJ, Haj-Hassan T, et al. Diagnostic value of laboratory tests in identifying serious infections in febrile children: systematic review. BMJ 2011;342:d3082.

29. Van den Bruel A, Jones C, Thompson M, et al. C-reactive protein point-of-care testing in acutely ill children: a mixed methods study in primary care. Arch Dis Child 2016;101:382-5.

30. Minnaard MC, van de Pol AC, de Groot JA, et al. The added diagnostic value of five different $\mathrm{C}$-reactive protein point-of-care test devices in detecting pneumonia in primary care: a nested case-control study. Scand J Clin Lab Invest 2015;75:291-5.

31. Prevention CfDCa. Clinical Laboratory Improvement Amendments (CLIA). Test Complexity. http://wwwn.cdc.gov/clia/resources/ testcomplexities.aspx. (accessed 20 Sep 2016).

32. Hardy V, Thompson M, Alto W, et al. Exploring the barriers and facilitators to use of point of care tests in family medicine clinics in the United States. BMC Fam Pract 2016;17:149.

33. Brown JB, Harris SB, Webster-Bogaert S, et al. Point-of-care testing in Diabetes Management: What Role Does It Play? Diabetes Spectr 2004;17:244-8.

34. Hobbs FD, Delaney BC, Fitzmaurice DA, et al. A review of near patient testing in primary care. Health Technol Assess 1997;1:i-iv, 1-229.

35. Barnett JM. Review. In: Benjamin Crabtree, William Miller ed. (1999). Doing Qualitative Research. 2nd edn. 2002;3 .

36. Howick J, Cals JW, Jones C, et al. Current and future use of point-of-care tests in primary care: an international survey in Australia, Belgium, The Netherlands, the UK and the USA. BMJ Open 2014;4:e005611.

37. Nesta. Longitude Prize 2014. Antibiotics. https:/www.nesta.org.uk sites/default/files/longitude-paper-antibiotics-open-review.pdf (accessed 20 Sep 2016).

38. ON J. Tackling drug-resistant infections globally. Final report and recommendations. 2016.

39. Weigl BH, Gaydos CA, Kost G, et al. The value of clinical needs assessments for point-of-care diagnostics. Point Care 2012;11:108-13.

40. Caliendo AM, Gilbert DN, Ginocchio CC, et al., Infectious Diseases Society of America (IDSA). Better tests, better care: improved diagnostics for infectious diseases. Clin Infect Dis 2013;57(Suppl 3): S139-70.

41. Holman RC, Curns AT, Singleton RJ, et al. Infectious disease hospitalizations among older American Indian and Alaska Native adults. Public Health Rep 2006;121:674-83. 